\title{
Simulation and measurement of high-Beta plasma in a magnetic nozzle
}

\author{
Christopher Deline $^{*}$ and Brian Gilchrist ${ }^{\dagger}$ \\ University of Michigan, Ann Arbor, MI 48109 \\ Roger Bengtson ${ }^{\ddagger}$ \\ University of Texas, Austin, TX 78712 \\ and \\ Jonathan Jones ${ }^{\S}$, Greg Chavers ${ }^{\S}$, and Chris Dobson ${ }^{\S}$ \\ NASA Marshall Space Flight Center, Huntsville, AL 35805
}

\begin{abstract}
The efficient extraction of thrust in a magnetic nozzle is of great importance to many future space propulsion designs. In several high-power EP thrusters, high magnetic fields are required to create and confine a quasineutral plasma. In order to produce thrust, this plasma exhaust must disengage from the applied magnetic fields downstream of the rocket. This paper presents a subset of results from an experiment investigating a high-powered plasma source emitting into a magnetic nozzle. In particular, numerical models and density profiles are presented which suggest that upon reaching high Beta (kinetic pressure > applied magnetic pressure) a flowing plasma will not be confined by the applied magnetic fields. The plasma will propagate as if it were detached from the magnetic fields.
\end{abstract}

\section{Nomenclature}

$\begin{array}{lll}B & = & \text { Magnetic field intensity } \\ \beta & = & \text { Ratio of plasma kinetic pressure to } \\ & \text { magnetic pressure } W_{k} / W_{B} \\ k & = & \text { Boltzmann's constant } \\ m_{i} & = & \text { Ion mass } \\ \mu_{0} & = & \text { Permeability of free space } \\ n_{e} & = & \text { Density of electrons } \\ n_{i} & = & \text { Density of ions } \\ n_{\max } & = & \text { Centerline plasma density for a } \\ N(z) & = & \text { Gaussian density distribution } \\ & & \end{array}$

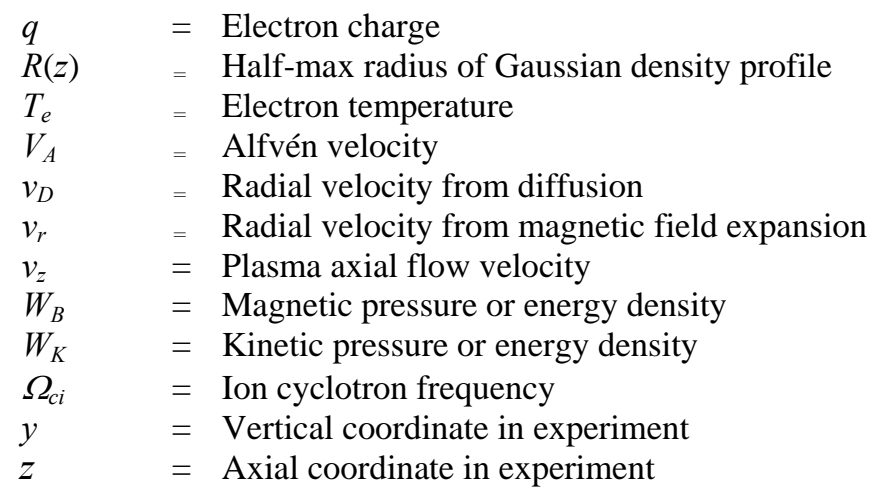

\section{Introduction}

EVERAL Electric Propulsion (EP) systems are being investigated which have high specific impulse and high $\checkmark$ power for future exploration missions. ${ }^{1-4}$ These propulsion systems utilize a plasma source aligned coaxially with a number of high strength (up to $1 \mathrm{~T}$ ) magnets, which shape and confine the plasma as it travels down the

\footnotetext{
* Graduate Student, AIAA Student Member cdeline@umich.edu

† Professor, Electrical Engineering and Space Systems, AIAA Associate Fellow

‡ Professor, Department of Physics, Fusion Research Center

$\S$ Physicist, NASA/MSFC/ER24
} 
axis of the rocket. One such propulsion system is the VAriable Specific Impulse Magnetoplasma Rocket $(\text { VASIMR })^{5}$, in which the plasma is also heated by a secondary RF power system and antenna that couple power to the plasma at the ion cyclotron frequency $\Omega_{c i}=q B / m_{i}$. The axial flow velocity $v_{z}$ of the plasma is further increased by the diverging magnetic field at the aft end of the rocket, due in part to the conservation of magnetic moment. This configuration forms a magnetic nozzle, which directs the plasma exhaust aft of the spacecraft without exposing material surfaces to the highly energetic exhaust plasma.

Several analyses ${ }^{6-10}$ predict that at sufficient distances downstream of the rocket, the plasma flow continues in the axial direction, detached from the influence of applied magnetic fields. In an applied magnetic nozzle, the plasma kinetic energy density $W_{k}=\frac{1}{2} m_{i} n_{i} v_{z}^{2}$ decreases downstream due to flux conservation. However, magnetic energy density $W_{B}=B^{2} / 2 \mu_{0}$ decreases more quickly and thus $\beta=W_{k} / W_{B}$, the ratio of the plasma's kinetic energy density to magnetic field energy density, will increase downstream. According to theory, when $\beta$ becomes greater than unity, a condition equivalent to the flow velocity exceeding the Alfvén velocity $V_{A}=B / \sqrt{\mu_{0} m_{i} n_{i}}$, the magnetized plasma becomes weakly self-magnetized with the external magnetic field no longer influencing the flowing plasma. This is a situation analogous to the solar wind flowing outward from the sun's magnetic field. ${ }^{11} \quad$ An experiment has been performed at the NASA Marshall Space Flight Center, Huntsville AL which has implemented a magnetic nozzle with diverging magnetic field lines ${ }^{12}$. Diagnostics were used to characterize the plasma flow along the length of the plume. Velocity, density and magnetic field data were some of the measurements taken as part of the experiment. This paper focuses mainly on the density profiles measured at various axial locations and compares them to theoretical predictions given a number of different detachment scenarios. In particular, three effects are modeled: plasma propagating purely along magnetic field lines, plasma diffusing across magnetic fields, and plasma detaching from magnetic field lines at high $\beta$. It is shown that it is the simulation accounting for all three effects which does the best job of predicting experimental results.

\section{Experiment Setup}

The experiments were conducted in the large $(2.75 \mathrm{~m}$ x $9 \mathrm{~m})$ vacuum chamber at the NASA Marshall Propulsion Research Center. A more detailed description of the experiment configuration for the Demonstration of Detachment Experiment (DDEX) has been presented previously. ${ }^{11,13}$ A top view of the experiment is given in Fig. 1 . The chamber uses current carrying coils to produce a small divergence angle magnetic nozzle with straight field lines. Two diffusion pumps allow the use of lighter gases (primarily hydrogen and helium) and keep the chamber base pressure below 10 microTorr. The chamber coordinate axis is arranged with axial position $z=0 \mathrm{~m}$ denoting the exit aperture of the magnetic nozzle.

External current carrying coils produce a magnetic nozzle with peak magnetic field intensity of $700 \mathrm{G}$ on-axis. Multiple large-diameter nozzle coil magnets are used in conjunction with a small bore, high-field choke magnet to produce straight diverging magnetic field lines up to $\mathrm{z}=0.8 \mathrm{~m}$. Magnetometer measurements on-axis confirm this field map.

Plasma was produced in a pulsed DC plasma washer gun ${ }^{14}$ operating up to $300 \mathrm{~kW}$. Data is presented using helium and hydrogen as a feedgas, with typical pulse lengths around a few milliseconds. Velocity measurements around $13 \mathrm{~km} / \mathrm{s}$ with Helium and $15 \mathrm{~km} / \mathrm{s}$ with Hydrogen were recorded using flux probes and Mach probes. An electron temperature of $2 \mathrm{eV}$ was found to reconcile the flux probe measurement to the Mach probe measurement for Hydrogen. Similarly, an electron temperature of $4 \mathrm{eV}$ was used for Helium data. The plasma pulse length of $2 \mathrm{~ms}$ allowed the plasma source to reach steady state.

\section{A. Measurements}

An X-Z position table inside the chamber allows radial scans of the plasma exhaust. A Langmuir triple-probe ${ }^{15}$ is positioned on the end of a boom, with cylindrical current collecting tips of diameter $0.09 \mathrm{~cm}$. This small Langmuir probe is oriented perpendicular to the plasma flow and allows fine spatial resolution scanning of the plume. Other instruments are mounted on the X-Z position boom, which are not considered in this paper. These instruments (Faraday probe, B-dot probes, Langmuir probe rake, Mach probe) are positioned hundreds of Debye lengths away from the Langmuir probe, far enough not to affect its readings. 
Additional plasma diagnostics include microwave interferometers which are positioned at three locations to provide spatial and temporal density measurements. A polychromatic quadrature interferometer is used which operates simultaneously at $70 \mathrm{GHz}, 90 \mathrm{GHz}$ and $110 \mathrm{GHz} .{ }^{16}$ This instrument is positioned near the nozzle entrance, $0.33 \mathrm{~m}$ downstream from the choke magnet. Plasma density measurements are taken via Langmuir probe just downstream of the chord of this interferometer. A second interferometer instrument is located at $\mathrm{z}=1.85 \mathrm{~m}$ at the nozzle exit aperture. This $15 \mathrm{GHz}$ quadrature interferometer ${ }^{17}$ provides a measurement resolution of at least $n_{e}=$ $10^{15} \mathrm{~m}^{-2}$ for line-integrated measurements along the $1.7 \mathrm{~m}$ beam length. Two independent interferometer chords are positioned at axial location $z=1.85 \mathrm{~m}$, with one chord on the axial centerline, and one positioned below it, at $y=$ $0.3 \mathrm{~m}$. Plasma density measurements were also taken via Langmuir probe along the centerline chord of this interferometer. At approximately $z=0.9 \mathrm{~m}$, the plasma $\beta$ is greater than unity, thus profile scans are accomplished both upstream and downstream of the expected detachment region.

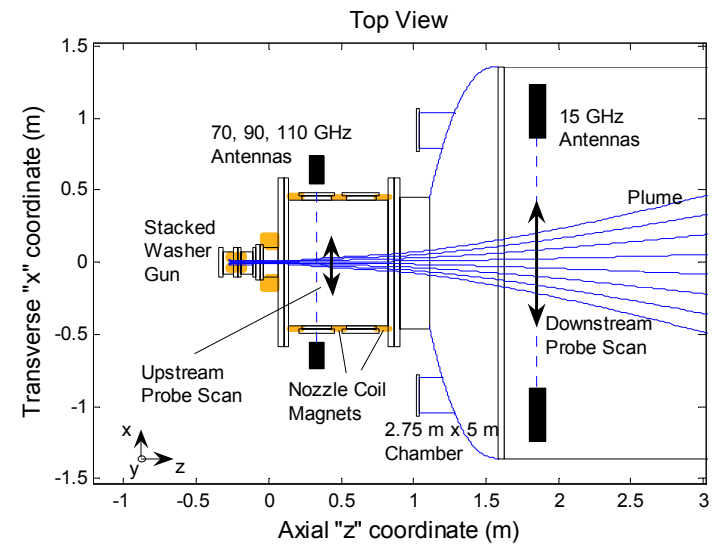

Figure 1: Top view of the detachment experiment setup. Upstream and downstream RF interferometers are shown along with radial Langmuir probe scan locations along the interferometer chords.

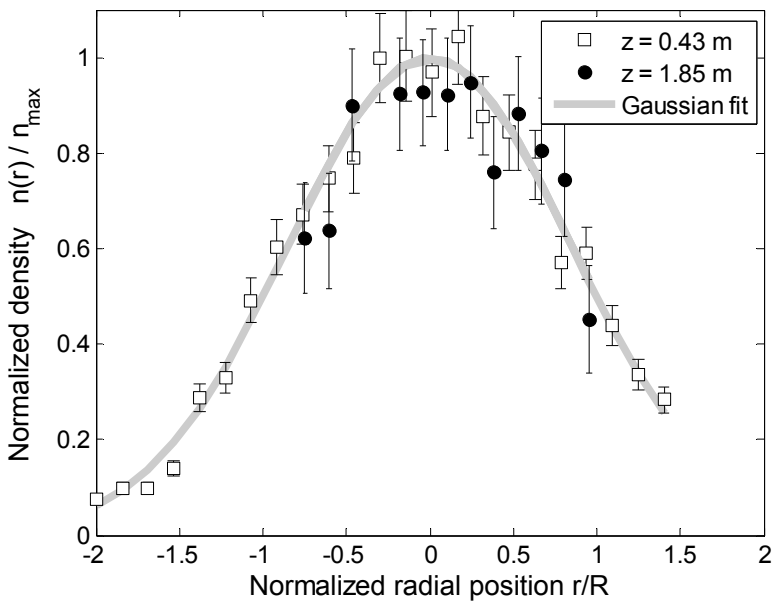

Figure 2: Upstream and downstream density profile for Hydrogen along the two interferometer chords. Gaussian least-squares fit shown for $z=0.43 \mathrm{~m}$ and $z=$ $1.85 \mathrm{~m}$. Peak density and column radius given in Table 1.

\section{Experiment Results}

The plasma radial profile was determined at a number of axial locations to determine the region in which the plume profile diverged significantly from the magnetic field profile. Two scanning techniques were used to determine the density profile, one involving scanning a microwave interferometer vertically across the plume and the other moving a Langmuir probe horizontally through the plume. Each was conducted at multiple axial locations, providing two independent measures of profile width.

\section{A. Langmuir probe scan and radial density distribution (Hydrogen and Helium)}

Accurate radial density profiles are produced by scanning the Langmuir triple-probe across the plasma column along a microwave interferometer chord. Shot to shot variation is factored out by normalizing each probe data point to the interferometer measurement for that shot. Probe measurements at various radial positions provide the shape of the plasma column. The interferometer chord density measurement is matched to the line integrated probe density measurement by introducing a scalar constant. This method takes advantage of the benefits of both instruments: accurate density measurements from an RF interferometer, and high spatial resolution measurements from an electrostatic probe. The radial profile of the plasma is thus accurately measured over several plasma shots and probe measurements. Probe scans were conducted at multiple axial locations: $\mathrm{z}=0.43 \mathrm{~m}, \mathrm{z}=0.88 \mathrm{~m}, \mathrm{z}=1.57$ $\mathrm{m}$ and $\mathrm{z}=1.85 \mathrm{~m}$ for Hydrogen, and $\mathrm{z}=0.43 \mathrm{~m}, \mathrm{z}=0.90 \mathrm{~m}$ and $\mathrm{z}=1.85 \mathrm{~m}$ for Helium.

As shown in Figure 2, the plasma profile for all radial scans can be fit to a Gaussian radial distribution.

$$
n(r, z)=n_{\max }(z) e^{\frac{-r^{2}}{\sigma^{2}}}=n_{\text {max }}(z) 2^{\frac{-r^{2}}{R(z)^{2}}}
$$


with $R(z)$ equal to the plasma half-max radius at position $z$ and $n_{\max }(z)$ equal to the centerline plasma density. (In this paper, the plasma radius at half-maximum is reported, not the more common 1/e radius). The goodness of fit was better for upstream measurements than for downstream measurements. This might partly be due to a change in profile shape where the plasma $\beta$ is high ( $>1$ ) as would be the case if the plume were to detach first at its high density core. The resulting centerline electron density and column width measured for each scan, averaged over the shot duration is given in the tables below:

\begin{tabular}{|l|l|l|l|l|}
\hline & $\mathrm{z}=0.43 \mathrm{H}$ & $\mathrm{z}=0.90 \mathrm{H}$ & $\mathrm{z}=1.57 \mathrm{H}$ & $\mathrm{z}=1.85 \mathrm{H}$ \\
\hline Density $\left(m^{-3}\right)$ & $3 \times 10^{18}$ & $6.4 \times 10^{17}$ & $1.2 \times 10^{17}$ & $1 \times 10^{17}$ \\
\hline$R(z)$ radius $(\mathrm{m})$ & 0.085 & 0.188 & 0.41 & 0.45 \\
\hline
\end{tabular}

Table 1: Scanning Langmuir probe results for Hydrogen plasma. Density reported is the mean centerline electron density for interferometer-calibrated Langmuir probe measurements. The profile width is reported as the plasma half-max radius.

\begin{tabular}{|l|l|l|l|}
\hline & $\mathrm{z}=0.43 \mathrm{He}$ & $\mathrm{z}=0.90 \mathrm{He}$ & $\mathrm{z}=1.85 \mathrm{He}$ \\
\hline Density $\left(m^{-3}\right)$ & $3.5 \times 10^{18}$ & $8 \times 10^{17}$ & $1 \times 10^{17}$ \\
\hline$R(z)$ radius $(\mathrm{m})$ & 0.095 & 0.19 & 0.50 \\
\hline
\end{tabular}

Table 2: Scanning Langmuir probe results for Helium plasma. Density reported is the mean centerline density for interferometer-calibrated Langmuir probe measurements. The profile width is reported as the plasma half-max radius.

Error estimates give an electron density uncertainty of $10 \%$ for the upstream scans $(\mathrm{z}=0.43 \mathrm{~m})$, and $20 \%$ for the remaining downstream scans. The plasma width error estimate was determined by Monte Carlo analysis; error bar values around $4-5 \%$ were calculated for the upstream scans $(z=0.43 \mathrm{~m})$ and $10-20 \%$ for select downstream scans.

\section{B. Interferometer scan and Abel inversion (Hydrogen)}

A scan of line integral density was produced at $z=0.33 \mathrm{~m}$ for the Hydrogen plume by manually adjusting the height of the upstream polychromatic interferometer from $y=+/-9 \mathrm{~cm}$ in $1.3 \mathrm{~cm}$ increments. The line integral density measurements were fit to a Gaussian distribution by least squares, and this analytic function subsequently used to determine radial electron density $n_{e}(r)$ via Abel inversion as discussed in Ref. 18. The resulting Gaussian radial profile is shown in Figure 3 and has a half-max radius of $0.05 \mathrm{~m}$ and a centerline density average of 1 x $10^{19}$ $\mathrm{m}^{-3}$. A downstream interferometer scan was also conducted at $\mathrm{z}=1.85 \mathrm{~m}$ by simultaneously measuring the two separate $15 \mathrm{GHz}$ interferometer chords. Assuming a Gaussian shaped profile the two chords define a density profile of radius $R(z)=0.45 \mathrm{~m}$, and peak electron density $\mathrm{n}_{\mathrm{e}}=1 \times 10^{17} \mathrm{~m}^{-3}$. Given an assumption of flux conservation, this suggests that the axial velocity is relatively constant (within $30 \%$ ) between the two interferometer positions. 


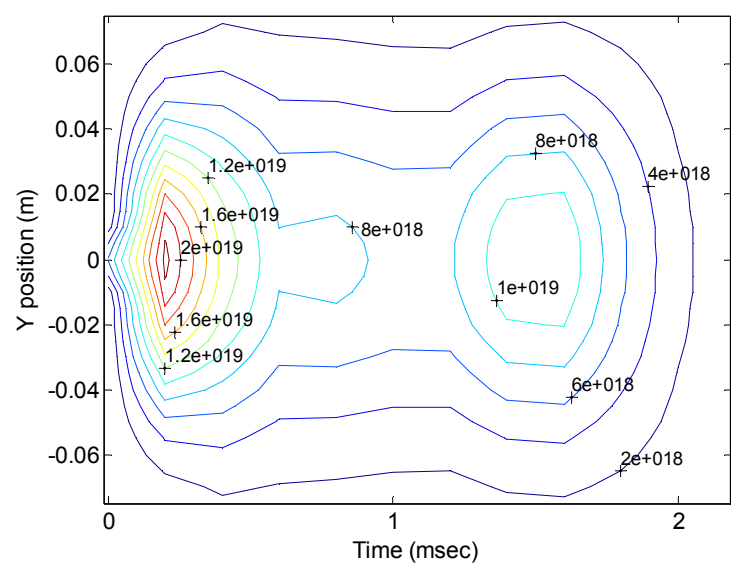

Figure 3: Scanning interferometer density profile for Hydrogen at $\mathbf{z}=\mathbf{0 . 3 3 m}$. Vertical interferometer scan showing electron density in $m^{-3}$ at $z=0.33 \mathrm{~m}$. Abel inversion of 48 separate measurements produced a Gaussian distribution with half-max radius of $R(z)=0.05$ $m$ and centerline density average of $1 \times 10^{19} \mathrm{~m}^{-3}$.

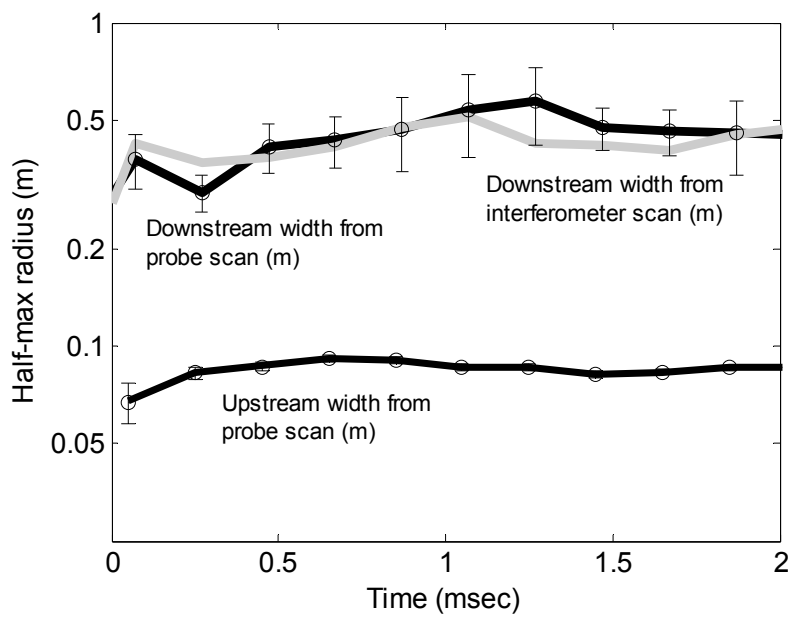

Figure 5: Column width measurement vs. time for Hydrogen. The upstream plume half-max radius $R(z)$ averaged 0.085 at $z=0.43 \mathrm{~m}$. The downstream plume $R(z)$ radius averaged $0.45 \mathrm{~m}$ at $z=1.85 \mathrm{~m}$ for both the probe scan and the interferometer scan. Plume widths determined by least-squares fit to a Gaussian distribution.

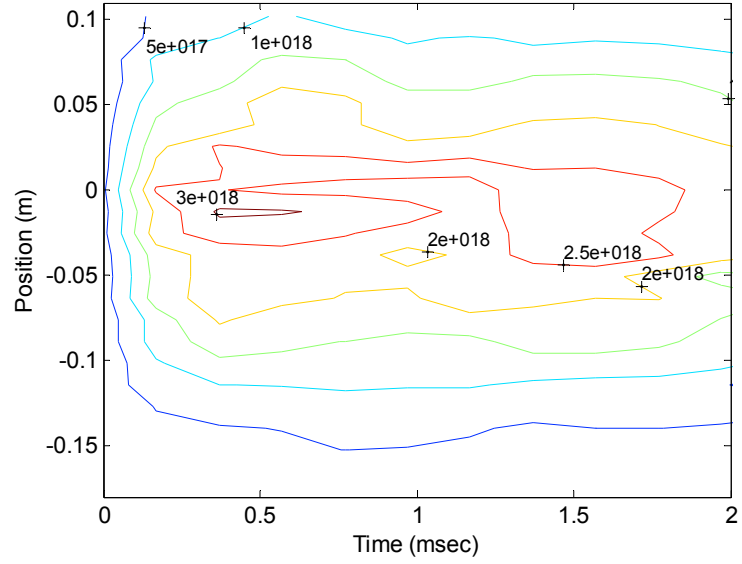

Figure 4: Langmuir Probe radial scan for Hydrogen at $\mathbf{z}=\mathbf{0 . 4 3 m}$.. Horizontal (probe) scan showing plasma density at $z=0.43 \mathrm{~m}$ from 23 separate shots. Centerline density: $3 \times 10^{18} \mathrm{~m}^{-3}$ with half-max radius of $R(z)=0.085 \mathrm{~m}$. Density error averaged $10 \%$ for this scan.

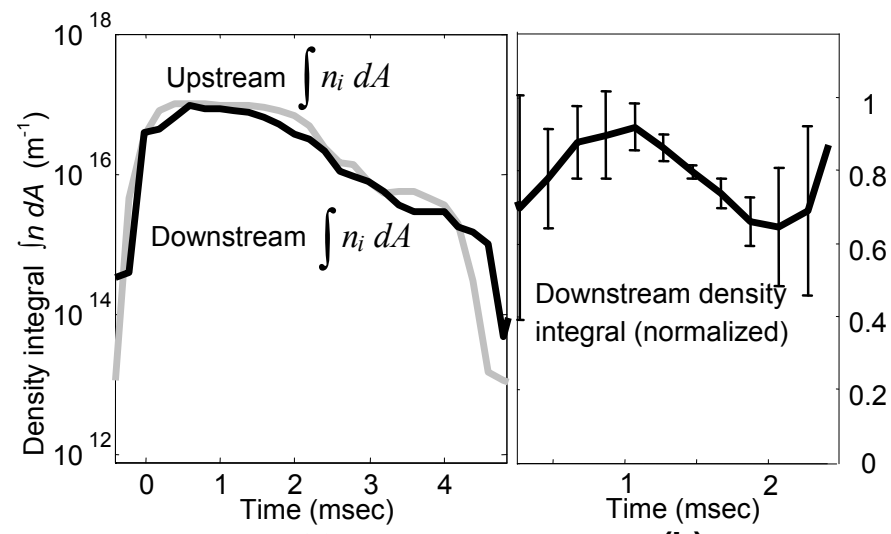

(a)

(b)

Figure 6: a): $\int n_{i} d A$ at two interferometer locations: $\mathbf{z}=$

$0.33 \mathrm{~m}$ and $\mathrm{z}=1.85 \mathrm{~m}$. Density integral determined from interferometer measurement and profile width. An estimate of $70 \%-90 \%$ ion flux conservation is given assuming constant velocity. $\mathbf{b}): \int n_{i} d A$ at $\mathbf{z}=\mathbf{1 . 8 5} \mathbf{m}$, normalized to upstream measurement. Area integral density is conserved within $30 \%$ for most of the shot with a decrease in density at the end of the shot. Collisional effects are implied. 


\section{Upstream / downstream density and flux conservation}

The continuity equation states that ion flux is conserved over two surfaces $A_{1}$ and $A_{2}$ perpendicular to the axis: $\int_{A_{1}} n_{i} q v_{z} d A=\int_{A_{2}} n_{i} q v_{z} d A$. Simultaneous interferometer measurements give a line integral of the electron density arriving at the two interferometer axial locations: $\mathrm{z}=0.33 \mathrm{~m}$ and $\mathrm{z}=1.85 \mathrm{~m}$. While the interferometers don't directly give area integral density, the aforementioned probe scans have provided profile shape and width information required to extract 2D area integrated density. Integrating Eq. (1) in 1D yields: $N(z)=\int_{-\infty}^{\infty} n_{\max }(z) 2^{\frac{-r^{2}}{R(z)^{2}}} d x=n_{\max } \sqrt{\pi(\ln 2)^{-1} R(z)^{2}}$ while integrating Eq. (1) in 2D over a surface perpendicular to the axis yields:

$$
\int n_{i} d A=\int_{0}^{2 \pi} \int_{0}^{\infty} n_{\max }(z) r 2^{\frac{-r^{2}}{R(z)^{2}}} d r d \phi=n_{\max } \pi(\ln 2)^{-1} R(z)^{2}=N(z) \sqrt{\pi(\ln 2)^{-1} R(z)^{2}}
$$

Thus area integral density is determined by $N(z)$ line integral density and $R(z)$ profile width, assuming axisymmetry and quasineutrality $\left(n_{i}=n_{e}\right)$.

By comparing $\int n_{i} d A$ at two axial locations, we can make one of two measurements: 1.) the acceleration of flow $\dot{v}_{z}$ assuming flux conservation, or 2 .) the degree of flux conservation assuming constant velocity $v_{z}$. As shown in Fig. 6, $\int n_{i} d A$ was 10\%-30\% lower at the downstream interferometer than at the upstream interferometer. A possible explanation for this decrease in downstream plasma density is the occurrence of collisions between ions and neutral gas, or plume impingement upon diagnostic probes and other structures in the plume. An alternative explanation is a $10-30 \%$ increase in flow velocity between the two positions. Since the flow velocity $v_{z}$ was not precisely known at the two interferometer positions, no definite distinction can be made between the two possibilities.

\section{Discussion}

\section{A. Plume trajectories}

Several models for plasma expansion were considered to account for the above radial profiles. These models were chosen to deal specifically with the measured plume diameters, given the assumption of constant velocity. The validity of this assumption as stated above is good within $\sim 20 \%$ for the beginning of a shot. The simplest initial model couples a static 2D vacuum magnetic field map with a steady state model in which the plume follows magnetic field lines exclusively. This is the case of a flowing plasma frozen onto vacuum field lines under steady state conditions. Additionally because the plume's radial density profile is approximately Gaussian at all locations, as stated in Eq. 1, a quasi-1D model was used in which the plume's $1 / 2$ max radius $R(z)$ is variable, but its density profile remains Gaussian. If the plasma plume is frozen onto magnetic field lines, the total captured magnetic flux $\pi R(z)^{2} B_{z}(z)$ is conserved, and the plume width is equal to $R(z) / R_{0}=\left(B_{z}(z) / B_{z, 0}\right)^{-1 / 2}$ where $R_{0}$ and $B_{z, 0}$ are the plasma $1 / 2$ max radius at $\mathrm{z}=0$ and magnetic field at $\mathrm{z}=0$, respectively. The radial expansion rate of the plume envelope $d R / d z$ can be defined by a radial velocity $v_{r}$ and a (constant) axial velocity $v_{z}$ where:

$$
\frac{d R(z)}{d z}=R_{0} B_{z, 0}{ }^{1 / 2} \frac{d}{d z}\left(B_{z}(z)\right)^{-1 / 2}=\frac{d R}{d t}\left(\frac{d z}{d t}\right)^{-1}=\frac{v_{r}}{v_{z}}
$$

A numerical simulation based on Euler's method is used with axial step size $d z=6.7 \mathrm{~mm}$, updating the profile width according to Eq. 2. Plasma density is calculated by flux conservation: $\frac{d}{d z}\left(R(z)^{2} n(z) v_{z}\right)=0$. Initial conditions were chosen with constant axial velocity $v_{z}$ equal to 1.5 times the sound speed, along with an initial electron density of $1 \times 10^{20} \mathrm{~m}^{-3}$ in the plasma source and an initial plume radius equal to $R_{0}=1.75 \mathrm{~cm}$ (less than the $2.5 \mathrm{~cm}$ radius of the plasma gun aperture). The profile width $R(z)$ is insensitive to the initial velocity, but the density is dependent on it. 
It was found that the radial expansion rate from this model is too slow to properly account for the measured plasma profiles. An improvement on the initial simulation includes the addition of a radial velocity term $v_{D}$ into Eq. 2 to account for cross-field diffusion:

$$
\frac{d R(z)}{d z}=\frac{v_{r}+v_{D}}{v_{z}}
$$

Here, $v_{D}$ is an approximate radial velocity due to diffusion at the plume's half-max radius. For the purposes of this simulation, radial magnetic field is ignored and $v_{D}$ is assumed to be exclusively radial. Furthermore, the rate of diffusion was chosen a posteriori to best fit the experimental data, thus this diffusion model can at best be considered a phenomenological one. A diffusion coefficient equal to or nearly equal to the Bohm diffusion coefficient was determined to best fit the experimental data; using Fick's law, $v_{D}$ can be defined from the Bohm diffusion coefficient: $v_{D}=-D_{B} \frac{\nabla n_{e}}{n_{e}}$ where $D_{B}=\frac{1}{16} \frac{k T_{e}}{B_{z}(R, z)}$ and $B_{z}(R, z)$ is the axial magnetic field strength calculated at $r=R(z)$. Using the Gaussian density profile from Eq. 1, the diffusion velocity reduces to:

$$
v_{D}=\frac{1}{16} \frac{k T_{e}}{B(r, z)} \frac{2 \ln 2}{R(z)^{2}}
$$

The same initial conditions and flux conservation assumptions that were used in the previous simulation are used again, with results given in Figs. 7 and 8. Best agreement with the Hydrogen data was found when the full diffusion velocity $v_{D}$ was used. Best agreement with the Helium data was found when a lower diffusion velocity equal to $v_{D} / 2$ was used.

A third trajectory model which also takes into account high- $\beta$ detachment appears to fit all measured profile widths. In this model, the ratio of plasma kinetic energy to magnetic field energy is tracked: $\beta=W_{k} / W_{B}=m n \mu_{0} v_{\|}{ }^{2} / B^{2}$. Once the plasma flow achieves $\beta>1$, the entire plume is assumed to detach at once, continuing on a ballistic trajectory i.e. $\frac{d^{2} R(z)}{d z^{2}}=0$. For both the Hydrogen and Helium simulations, $\beta$ reaches unity at approximately $z=0.9 \mathrm{~m}$.

As can be seen in Figs 7 and 8, only assuming strict field line scaling will underestimate the plume width by a significant amount. The improved cross-field diffusion model results in the upstream radial density profiles being properly accounted for, but not the downstream density profiles. The diffusion rate could be arbitrarily set to a lower value in attempts to better fit the downstream density profiles. But in this case, the upstream trajectories would not fit. The high- $\beta$ detachment trajectory model best accounts for both the upstream and downstream column widths measured in the experiment. 


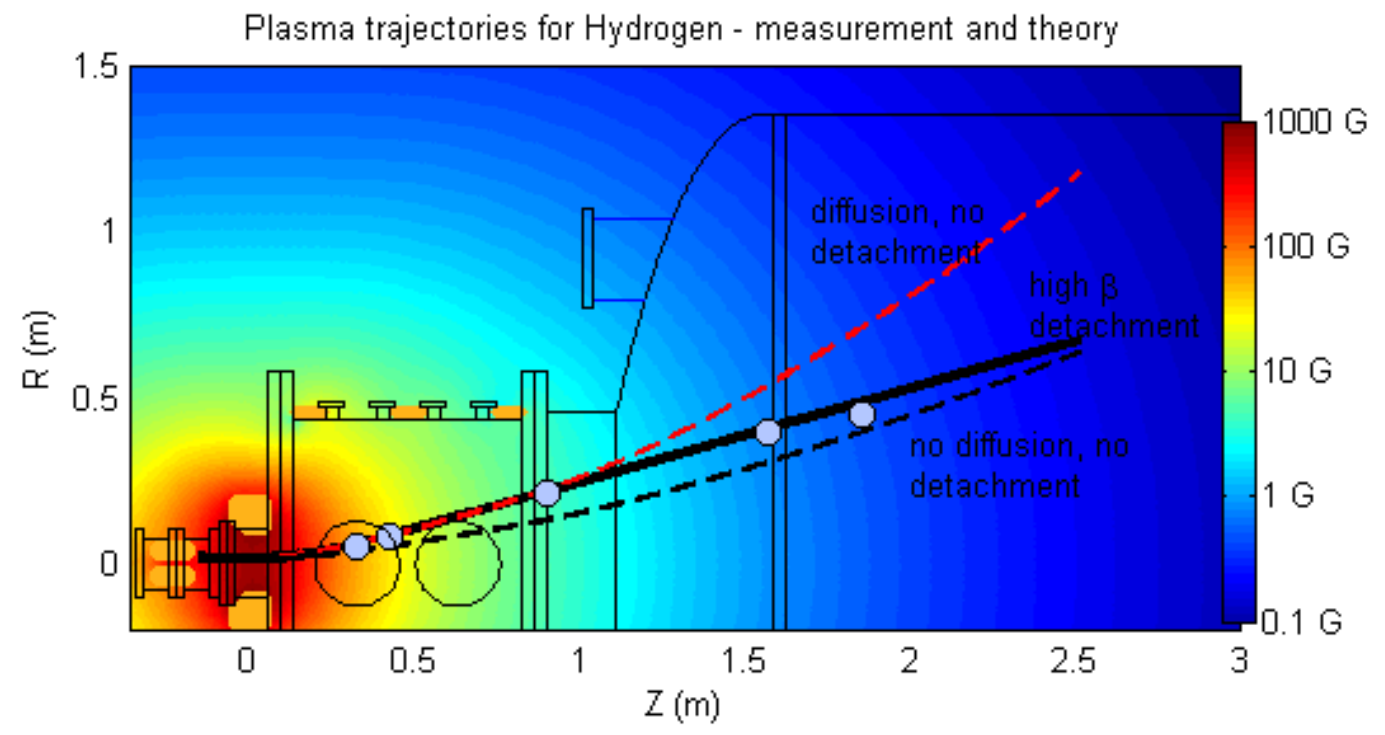

Figure 7: Plume trajectories for Hydrogen. $T_{e}=2 \mathrm{eV}$. Black dashed line shows field line mapping only. Red dashed line shows field line mapping plus Bohm cross-field diffusion. Black solid line shows field line mapping, plus cross-field diffusion, plus $\beta>1$ detachment. Diffusion velocity $v_{D}$ was set equal to Bohm diffusion rate. Dots represent measured results from Table 1.

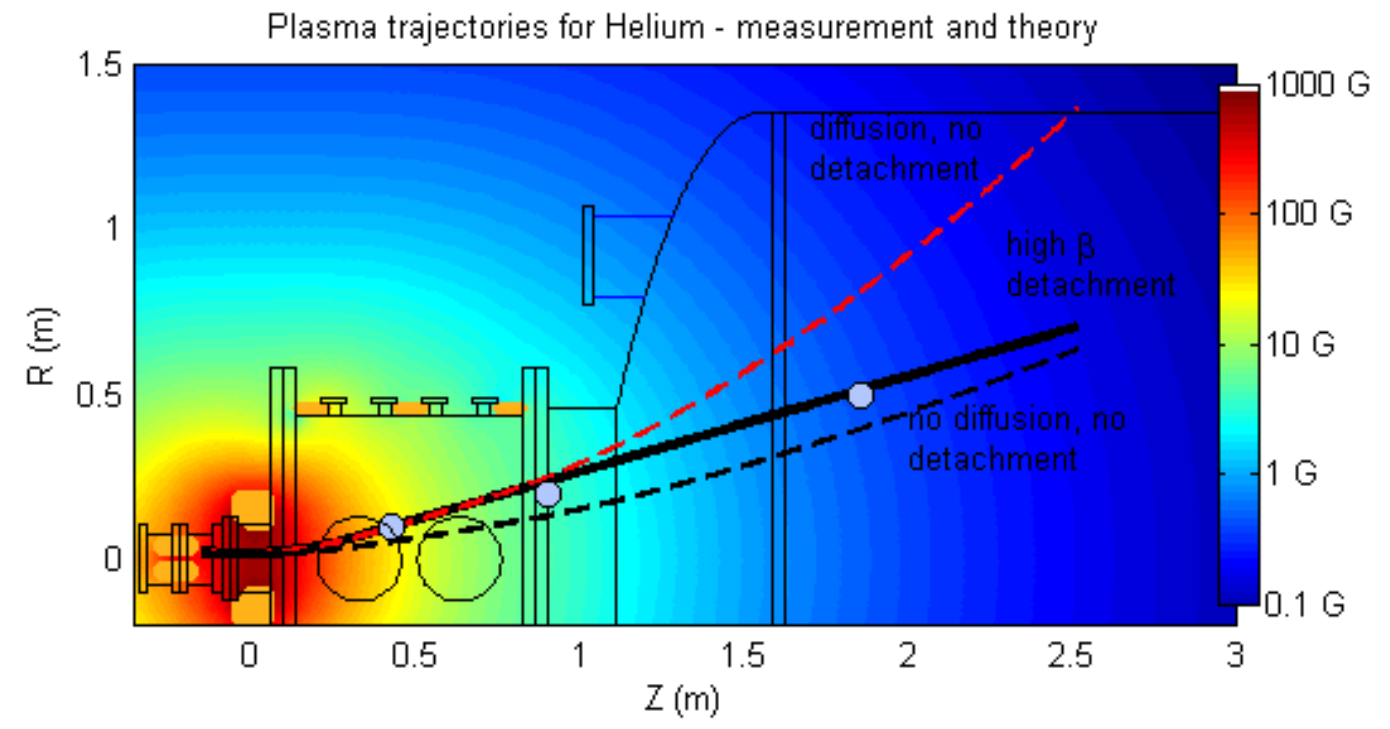

Figure 8: Plume trajectories for Helium. $T_{e}=4 \mathrm{eV}$. Black dashed line shows field line mapping only. Red dashed line shows field line mapping plus Bohm cross-field diffusion. Black solid line shows field line mapping, plus crossfield diffusion, plus $\beta>1$ detachment. Diffusion velocity $v_{D}$ was set equal to Bohm/2 diffusion rate. Dots represent measured results from Table 2.

\section{B. Further work}

The measurements and simulations given in this paper are only part of a larger campaign concerned with the physics of magnetic nozzles. Velocity data is not considered here, and the simulations used to calculate trajectories do not incorporate more advanced MHD code. In order to gain a more complete picture of what is happening in this experiment, these effects should be considered. In fact, a more complete MHD code has been developed which 
includes plasma magnetic fields in addition to vacuum magnetic fields. The results of this code have been accepted for publication ${ }^{19}$, and differ only slightly from the results presented here.

The models used in this paper to simulate the trajectories are simple, and neglect some effects which may play a role in the physics of magnetic detachment. One important effect not considered by this model is a change in the plume profile shape. The quasi-1D approximation considered here requires the profile to stay the same, thus requiring the plume to detach as a whole. A more likely scenario of detachment would involve a detachment contour, with the high density core detaching first and the lower density outer edge following. This may have some effect on the overall nozzle efficiency, and may lead to a tenuous layer of plasma being scraped off from the plume. A full MHD simulation would be able to predict this occurrence. The second effect not considered in this paper is a variable velocity. While comparison of upstream density and downstream density measurements suggests that constant velocity is a reasonable approximation for this experiment, knowing the flow velocity and momentum flux is a requirement for any estimate of nozzle efficiency or acceleration due to the expanding magnetic field. Further work will be done to present these results.

\section{Summary}

A high power $(300 \mathrm{~kW})$ pulsed light gas plasma source was integrated with a magnetic nozzle in the DDEX experiment at NASA MSFC. The plasma flow was characterized both upstream in the low- $\beta$ region, and downstream in the high- $\beta$ region. Radial density profiles were measured at various axial locations using two separate microwave interferometer devices, and a translation stage scanning Langmuir probe. The plasma profiles were compared with a quasi-1D simulation that accounted for plume expansion along field lines, radial diffusion across field lines, and detachment from field lines at high $\beta$.

The measured plasma density profiles could not be explained by strict field-line mapping of density, but it appears that a model including Bohm cross-field diffusion and detachment from field lines at the point of $\beta$ reaching unity does help to explain the profile widths. Although direct measurement of flow velocity was not presented in this paper, constant velocity or slight acceleration would be consistent with measurements of density conservation between the upstream and downstream interferometer location. Additionally, plasma exhaust appeared to flow across the $\beta=1$ boundary without significant perturbation, and continued as if it were following ballistic trajectories. Even at a distance of several meters, a high percentage of area integrated plasma density arrived at the downstream interferometer with no indication of a decrease in flow velocity. This shows the potential for good nozzle efficiency on proposed high-power, high-magnetic field plasma rockets such as VASIMR, and suggests that a properly designed magnetic nozzle can lead to a significant portion of the plasma exhaust detaching from applied magnetic fields.

\section{Acknowledgments}

This work was supported in part by NASA contract NNJ05HB77C and by a NASA GSRP fellowship. Special thanks go to the UT Austin team including Boris N. Breizman, to Adrian Lopez at the University of Michigan and to Mark Carter and Andrew Ilin at the Ad Astra Rocket Company.

\section{References}

\footnotetext{
${ }^{1}$ F.R. Chang Diaz, “Research Status of the Variable Specific Impulse Magnetoplasma Rocket”, Fusion Technology, Vol. 35, No. 1T, 1999 pp. 87-93

${ }^{2}$ C. Charles, R.W. Boswell, “Current-Free Double-Layer Formation in a High-Density Helicon Discharge,” Applied Physics Letters, Vol. 82, No. 9, 2003

3 J.Gilland, “Application of a Helicon Discharge to Electric Propulsion” AIAA paper 1998-3934, July 1998.

${ }^{4}$ V.B. Tikhonov, S.A. Semenikhin, J.R. Brophy, and J.E.Polk, "Performance of $130 \mathrm{~kW}$ MPD Thruster with an External Magnetic Field and Li as Propellant”, International Electric Propulsion Conf., paper 97-117, 1997

${ }^{5}$ A.Arefiev, B.Breizman, “Theoretical Components of the VASIMR propulsion concept” Physics of Plasmas, Vol. 11, No. 5, 2004

${ }^{6}$ A.V. Arefiev, B.N. Breizman, “Magnetohydrodynamic Scenario of Plasma Detachment in a Magnetic Nozzle” Physics of Plasmas, Vol. 12, No. 4, 2005
} 
${ }^{7}$ A.V. Ilin, F.R.Chang-Diaz, J.P. Squire, A.G. Tarditi, B.N. Breizman, M.D. Carter, "Simulations of Plasma Detachment in VASIMR,” AIAA Paper 2002-0346, Jan. 2002

${ }^{8}$ F.N. Gesto, B.D. Blackwell, C.Charles, R.W. Boswell, "Ion detachment in the helicon double-layer thruster exhaust beam” Journal of Propulsion and Power, Vol. 22, No. 1, 2006

${ }^{9}$ N. Brenning, T. Hurtig, M.A. Raadu, “Conditions for plasmoid penetration across abrupt magnetic barriers”, Physics of Plasmas, Vol. 12, No. 1, 2005

10 J.T. Cassibry, “Theoretical performance of an MHD nozzle using super-Alfvenic detachment”, AIAA paper 2006-5160, July 2006

${ }^{11}$ E.N. Parker, “Dynamics of the interplanetary gas and magnetic fields”, Astrophysical Journal, Vol. 128, No. 3, 1958, pp. 664-676

${ }^{12}$ G. Chavers et al., "Status of magnetic nozzle and plasma detachment experiment”, AIAA Space Technology and Applications International Forum, February 2006

${ }^{13}$ C. Deline, D.G. Chavers, B. Gilchrist, "Physics of Plasma Detachment in a Magnetic Nozzle” AIAA paper 2006-4653, July 2006

${ }^{14}$ G. Fiksel, A.F. Almagri, D. Craig, M. Lida, S.C. Prager, J.S. Sarff, "High current plasma electron emitter” Plasma Sources Science and Technology, Vol. 5, 1996 pp. 78-83

${ }^{15}$ S. Buffton, "Exit plane plasma measurements of a low-power hydrazine arcjet”, PhD Thesis, University of Illinois, Urbana-Champaign 1996

${ }^{16}$ C. Dobson, J. Jones, D.G. Chavers, "Instrument reflections and scene amplitude modulation in a polychromatic microwave quadrature interferometer” Review of Scientific Instruments, Vol. 75, No. 3, 2004

${ }^{17}$ B. Gilchrist, S. Ohler, A. Gallimore, "Flexible microwave system to measure the electron number density and quantify the communications impact of electric thruster plasma plumes”, Review of Scientific Instruments, Vol. 68, No. 2, 1997

${ }^{18}$ I.H. Hutchinson, Principles of Plasma Diagnostics, University Press, Cambridge, 2002

${ }^{19}$ B.N. Breizman, et al., Physics of Plasmas, 2007 (to be published) 